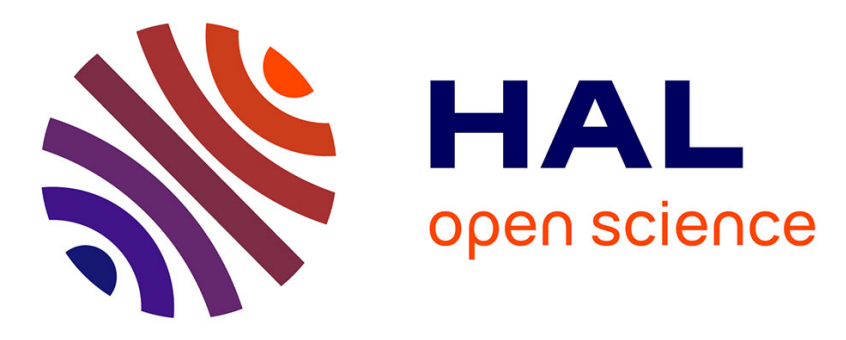

\title{
Precoding and Decoding in the MIMO Interference Channel for Discrete Constellation
}

\author{
Yasser Fadlallah, Amir Khandani, Karine Amis Cavalec, Abdeldjalil Aissa El \\ Bey, Ramesh Pyndiah
}

\section{- To cite this version:}

Yasser Fadlallah, Amir Khandani, Karine Amis Cavalec, Abdeldjalil Aissa El Bey, Ramesh Pyndiah. Precoding and Decoding in the MIMO Interference Channel for Discrete Constellation. PIMRC 2013: IEEE International Symposium on Personal, Indoor and Mobile Radio Communications, Sep 2013, Londres, United Kingdom. pp.1157 - 1161. hal-00931029

\section{HAL Id: hal-00931029 \\ https://hal.science/hal-00931029}

Submitted on 14 Jan 2014

HAL is a multi-disciplinary open access archive for the deposit and dissemination of scientific research documents, whether they are published or not. The documents may come from teaching and research institutions in France or abroad, or from public or private research centers.
L'archive ouverte pluridisciplinaire HAL, est destinée au dépôt et à la diffusion de documents scientifiques de niveau recherche, publiés ou non, émanant des établissements d'enseignement et de recherche français ou étrangers, des laboratoires publics ou privés. 


\title{
Precoding and Decoding in the MIMO Interference Channels for Discrete Constellation
}

\author{
Yasser Fadlallah ${ }^{1,2}$, Amir Khandani ${ }^{2}$, Karine Amis ${ }^{1}$, Abdeldjalil Aïssa-El-Bey ${ }^{1}$, and Ramesh Pyndiah ${ }^{1}$ \\ ${ }^{1}$ Institut Télécom; Télécom Bretagne; UMR CNRS 6285 Lab-STICC \\ Technopôle Brest Iroise CS 8381829238 Brest, France \\ Université européenne de Bretagne \\ ${ }^{2}$ University of Waterloo \\ Email: Firstname.Lastname@telecom-bretagne.eu
}

\begin{abstract}
This paper addresses the problem of decoding and precoding in the K-user MIMO interference channels. At the receiver side, a joint decoding of the interference and the desired signal is able to improve the receive diversity order. At the transmitter side, we introduce a joint linear precoding design that maximizes the joint cut-off rate, known as a tight lower bound on the joint mutual information for high signal-to-noise ratio (SNR). We also derive a closed-form solution of the precoding matrices that maximizes the mutual information when the SNR is close to zero. This solution is characterized by its low computational complexity, and only requires a local channel state information knowledge at the transmitters. Our simulation results show that decoding interference jointly with the desired signal results in a significant improvement of the receive diversity order. Also a substantial bit error rate and sum-rate improvements are illustrated using the proposed precoding designs.
\end{abstract}

\section{INTRODUCTION}

Recently, an intense focus of research has been steered toward the transmission in multiple-input multiple-output (MIMO) interference channel (IC). The asymptotic behavior of its capacity has been defined using joint linear precoding [1], [2]. The idea of linear precoding has been basically introduced for additional white Gaussian noise (AWGN) channel, with the goal of minimizing the weighted mean squared error [3]. This idea has been extended to the single user MIMO channel. It has been proven that linear precoding is able to achieve the channel capacity when the full CSI is provided at the transmitter [4]. Later on, the authors in [2], [5] have shown that joint linear precoding design can also achieve the asymptotic capacity of the MIMO IC. This joint precoding design is known as interference alignment (IA).

The basic idea of IA is to jointly design the signals from all transmitters such that interfering signals at each receiver overlap and remain distinct from the desired signal. At the receiver side, a zero forcing $(\mathrm{ZF})$ decoder is traditionally applied to cancel the aligned interference [1], [6]. However, the approach of canceling the interference using $\mathrm{ZF}$ results in a receive diversity loss. In contrast, decoding the interference jointly with the desired signal can improve the receive diversity order, as shown hereafter.

In this paper, we firstly discuss the achievable receive diversity of both schemes: interference decoding and traditional interference cancellation. Secondly, we aim to find out an efficient linear precoding design assuming a discrete constellation. The linear precoding scheme that maximizes the Mutual Information (MI) for discrete constellation in a single user MIMO channel has been addressed in [7]. The authors have proved that the MI is a concave function of a matrix which is itself a quadratic function of the precoding matrix. In our work, we address the linear precoding in the multi-user MIMO IC. We do not aim to maximize the MI, but rather to maximize the joint cut-off rate between all transmitted signals. The joint cut-off rate has two characterizations:

1) it is defined as a tight lower bound on the joint mutual information for high signal-to-noise ratio (SNR),

2) it can be expressed analytically, and does not require a numerical integration.

We also propose a closed form solution for precoding matrices that maximizes the MI when the signal-to-noise ratio (SNR) tends to zero. It is obtained using the first order Taylor expansion of the MI at very low SNR [8]. It is mainly characterized by its low computational complexity, and only requires local channel state information at the transmitters (CSIT).

This paper is organized as follows. In Section II, we describe the system model of the multi-user MIMO IC. In Section III, we compare the achievable receive diversity for both schemes traditional interference cancellation and interference decoding. Then we formulate the joint cutoff rate in Section IV, and we propose an iterative algorithm that converges toward a local optimal solution. The closed form solution is given in Section V. In Section VI, the simulation results evaluate our contributions. Finally, Section VII concludes the paper.

Notations: boldface upper case letters and boldface lower case letters denote matrices and vectors, respectively. For the transpose, transpose conjugate and conjugate matrix we use $(.)^{T},(.)^{H}$ and $(.)^{*}$, respectively. $\|\|,. \operatorname{tr}($.$) and \log$ stand for the Frobenius norm, the trace operator and $\log _{2}$. $\mathbb{E}$ is the expectation operator.

\section{SYSTEM MODEL}

Consider a $K$-user MIMO IC, with equal number of antennas $N_{t}$ at the transmitters and equal number of antennas $N_{r}$ at the receivers. The $K$ transmit-receive pairs share a common channel, where each transmitter intends to have its signal 
decoded by its destination. The symbols of the original signal vector at transmitter $j$, denoted $x_{j}$ with dimension $d_{j} \times 1$, are selected with equal probabilities from a discrete constellation $\mathcal{Q}$ with size $M$ shared by all transmitters. The original signal vector is then precoded using linear transformation matrix $\boldsymbol{P}_{j}$ with dimensions $N_{t} \times d_{j}$. The $k^{t h}$ received signal is given by

$$
\boldsymbol{y}_{k}=\sum_{j=1}^{K} \boldsymbol{H}_{k j} \boldsymbol{P}_{j} \boldsymbol{x}_{j}+\boldsymbol{n}_{k}
$$

where $\boldsymbol{H}_{k j} \in \mathbb{C}^{N_{r} \times N_{t}}$ is the channel matrix between the $j^{\text {th }}$ transmitter and the $k^{\text {th }}$ receiver, and $\boldsymbol{n}_{k} \in \mathbb{C}^{N_{r}}$ is the circularly symmetric additive Gaussian noise vector with zero mean and covariance $\sigma^{2} \boldsymbol{I}_{N_{r}}$, i.e. $\boldsymbol{n}_{k} \sim N\left(0, \sigma^{2} \boldsymbol{I}_{N_{r}}\right)$. In the following, we assume that $N_{t}=N_{r}$.

Using IA design at the transmitters, where the precoders are jointly designed to align interference at all receivers [6], the received signal can be decomposed in two linearly independent subspaces as

$$
\boldsymbol{y}_{k}=\underbrace{\boldsymbol{H}_{k k} \boldsymbol{P}_{k}}_{\text {Desired subspace }} \cdot \boldsymbol{x}_{k}+\underbrace{\sum_{j \neq k} \boldsymbol{H}_{k j} \boldsymbol{P}_{j}}_{\text {interference subspace }} . \boldsymbol{x}_{j}+\boldsymbol{n}_{k} .
$$

The traditional decoding method consists in canceling the interference using linear $\mathrm{ZF}$, and yields

$$
\begin{aligned}
\hat{\boldsymbol{y}}_{k}=\boldsymbol{U}_{k}^{0^{H}} \boldsymbol{y}_{k} & =\boldsymbol{U}_{k}^{0^{H}} \boldsymbol{H}_{k k} \boldsymbol{P}_{k} \boldsymbol{x}_{k}+\boldsymbol{U}_{k}^{0^{H}} \boldsymbol{n}_{k} \\
& =\overline{\boldsymbol{H}}_{k} \boldsymbol{x}+\boldsymbol{U}_{k}^{0^{H}} \boldsymbol{n}_{k},
\end{aligned}
$$

where the $\hat{\boldsymbol{y}}$ is the $d_{k} \times 1$ free-interference signal vector, $\boldsymbol{U}_{k}^{0^{H}}$ is the interference canceler defined in (8), and $\overline{\boldsymbol{H}}_{k}=\boldsymbol{U}_{k}^{0^{H}} \boldsymbol{H}_{k} \boldsymbol{P}_{k}$ with dimensions $d_{k} \times d_{k}$. Another decoding method known as interference decoding, where the receiver decodes jointly the desired and the undesired signals and keeps only the desired part. In this case, the received signal can be rewritten as

$$
\begin{aligned}
\hat{\boldsymbol{y}}_{k} & =\left(\boldsymbol{H}_{k 1} \boldsymbol{P}_{1}, \cdots, \boldsymbol{H}_{k K} \boldsymbol{P}_{K}\right)\left(\begin{array}{c}
\boldsymbol{x}_{1} \\
\vdots \\
\boldsymbol{x}_{K}
\end{array}\right)+\boldsymbol{n}_{k}, \\
& =\overline{\boldsymbol{H}}_{k} \boldsymbol{x}+\boldsymbol{n}_{k}
\end{aligned}
$$

where $\overline{\boldsymbol{H}}_{k}$ is the new channel matrix with dimensions $N_{r} \times$ $\sum_{j=1}^{K} d_{j}$.

In order to decode the original information in (3) and (4), we propose a joint minimum-distance (MD) detector. The MD detector involves an exhaustive search over all possible transmitted vectors. The signal is decoded as

$$
\hat{\boldsymbol{x}}=\underset{i}{\arg \min }\left\|\hat{\boldsymbol{y}}_{k}-\overline{\boldsymbol{H}}_{k} \boldsymbol{x}^{i}\right\|^{2},
$$

where $\hat{\boldsymbol{y}}_{k}$ is equivalent to (3) ((4)) when the first (second) decoding method is applied, and $i$ is over all possible transmitted vectors $\boldsymbol{x}$ in (3) and (4). In the next section, the receive diversity order of both aforementioned decoding schemes is discussed.

\section{RECEIVE DIVERSITY ORDER}

As mentioned in the previous section, our channel model can be seen as a single user MIMO channel in both cases: the $\mathrm{ZF}$ interference canceler and the MD-interference decoder. For a single user MIMO channel, the generic receiver equation is equal to

$$
\boldsymbol{y}_{D_{R}}=\boldsymbol{H}_{D_{R} \times D_{T}} \boldsymbol{x}_{D_{T} \times 1}+\boldsymbol{n}_{D_{R}} \text {. }
$$

Assuming an MD decoder, the probability of error can be approximated at high SNR by [9]

$$
P_{e}=\alpha \mathrm{SNR}^{-D_{T} D_{R}},
$$

where $\alpha$ points out the horizontal shift of the $P_{e}$ curve, $D_{T}$ is the transmit diversity gain, and $D_{R}$ is the receive diversity gain. Assuming $D_{T}=1$, (7) indicates that the slope of the probability of error is proportional to the inverse of SNR to the power $D_{R}$. $D_{R}$ is equal to the number of independent observations at the receiver, and remains independent of the number of transmit antennas. This result has been established in [10], where the authors have concluded that using an MD detector, only an SNR penalty is introduced when the number of transmit antennas increases. In the following, an analogy between the single user MIMO channel and both channel models given in (3) and (4) is presented for the purpose of showing the expected receive diversity of each decoding scheme.

\section{A. Interference cancellation using zero forcing}

In order to cancel the interference, the received signal can be projected onto the interference null space as shown in (3). The projection matrix at receiver $k$ is given using the singular value decomposition of the interference subspace as

$$
\boldsymbol{U}_{k} \boldsymbol{S}_{k} \boldsymbol{V}_{k}=\sum_{j \neq k} \boldsymbol{H}_{k j} \boldsymbol{P}_{j}
$$

where $\boldsymbol{U}_{k}=\left[\boldsymbol{U}_{k}^{1}, \boldsymbol{U}_{k}^{0}\right]$ consists of the interference space $\boldsymbol{U}_{k}^{1} \in \mathbb{C}^{N_{r} \times\left(N_{r}-d_{k}\right)}$ and the interference null space $\boldsymbol{U}_{k}^{0} \in$ $\mathbb{C}^{N_{r} \times d_{k}}$,respectively. Projecting the received signal on $\boldsymbol{U}_{k}^{0}$, the new channel model is obtained as in (3). This model yields a $d_{k} \times d_{k}$ MIMO single user channel. Hence, (7) implies that the expected receive diversity order $D_{R}$ is equivalent to $d_{k}$, describing the free-interference subspace dimensions.

\section{B. Interference decoding scheme}

On the other hand, accounting the interference incurred from other users as a desired signal, the new channel model remains as in (4). This channel model can be assumed as an $N_{r} \times\left(\sum_{j=1}^{K} d_{j}\right)$ MIMO single user channel. The signal $\left[\boldsymbol{x}_{1}^{T}, \cdots, \boldsymbol{x}_{K}^{T}\right]^{T}$ composed of the desired signal and the interference is jointly decoded using $N_{r}$ independent observations. Hence, the expected receive diversity gain achieved with an MD detector, is $D_{R}=N_{r}$. Consquently, as long as $d_{k} \leq N_{r}$, a higher diversity can be achieved using the MD-interference decoding scheme at each receiver.

Remark 1: The receive diversity does not depend on the precoding scheme, but rather on the number of observations at 
the receiver. Therefore, in the upcoming sections we consider the MD-interfernce decoding receiver that results in a higher receive diversity, and we try to optimize the precoding matrices so as to maximize the joint cut-off rate and the MI.

\section{Precoding MATRIX OPtimization}

The linear precoding optimization problem based on the maximization of the MI has been exploited in many papers for both cases: canceling interference in an IA scheme, and treating interference as noise [11]-[13]. The MI has been maximized under the assumption of Gaussian input distribution. However, this assumption seems to be far away from the practical systems that employ a discrete constellation such as phase shift keying (PSK) modulation and quadratic amplitude modulation (QAM). Under the discrete constellation assumption, one can propose to maximize the MI in the IC between each transmitter-receiver pair. However, it is not obvious whether the MI for discrete constellation can be expressed in closed form or not, and its computation requires numerical integration that becomes intractable with increase of the signal and constellation dimensions. On the other side, a closed form is available for the joint cut-off rate ${ }^{1}$ that represents a lower bound on the MI that becomes very tight when the SNR increase. Therefore, we try to maximize the joint cut-off rate subject to the transmit power constraint trace $\left(\boldsymbol{P}_{k}^{H} \boldsymbol{P}_{k}\right) \leq d_{k}$ for all $k$. As mentioned above, one of the advantages of this criterion is that it can be expressed analytically without the need of a numerical integration. For the sake of simplicity, we assume an equal power allocation at all transmitters. For a uniform inputs distribution, the joint cut-off rate between the $k^{\text {th }}$ receiver and the transmitters can be mathematically expressed as [16], [17]

$$
\begin{aligned}
R_{0_{k}} & =-\log \left[\int_{\boldsymbol{y}_{k}}\left(\sum_{i} \sqrt{p\left(\boldsymbol{y}_{k} \mid \boldsymbol{x}^{i}\right)}\right)^{2} \mathrm{~d} \boldsymbol{y}_{k}\right] \\
& =-\log \left[\int_{\boldsymbol{y}_{k}}\left(\sum_{i, j} \sqrt{p\left(\boldsymbol{y}_{k} \mid \boldsymbol{x}^{i}\right) \cdot p\left(\boldsymbol{y} \mid \boldsymbol{x}^{j}\right)}\right) \mathrm{d} \boldsymbol{y}_{k}\right] \\
& \stackrel{a}{=}-\log \sum_{A, A^{\prime}} \exp \left(-p \frac{\left\|\sum_{j=1}^{K} \boldsymbol{H}_{k j} \boldsymbol{P}_{j}\left(\boldsymbol{x}_{j}^{a_{j}}-\boldsymbol{x}_{j}^{a_{j}^{\prime}}\right)\right\|^{2}}{4 \sigma^{2}}\right)
\end{aligned}
$$

where $p$ is the transmit power, $A^{\prime}=\left\{a_{1}^{\prime}, \cdots, a_{K}^{\prime} \mid a_{j}^{\prime} \in\right.$ $\left.\mathcal{M}_{d_{j}}, \forall j\right\}$ and $A=\left\{a_{1}, \cdots, a_{K} \mid a_{j} \in \mathcal{M}_{d_{j}}, \forall j\right\}$. The last step $a$ is straightforward using the fact that $\boldsymbol{y}_{k}$ is corrupted by a zero mean Gaussian noise, i.e. $\boldsymbol{n}_{k} \sim \mathcal{N}_{c}\left(0, \sigma^{2} \boldsymbol{I}_{N_{r}}\right)$ ( [18] example 2.6.2). It is clear from (9) that no numerical integration is required. The maximization problem of the sum of all joint cut-off rates in the $K$-user MIMO IC can be achieved using the iterative algorithm given below. In this algorithm, the gradient of the sum of $R_{0}$ over all users is

\footnotetext{
${ }^{1}$ The cut-off rate, symbolized by $R_{0}$, characterizes a lower bound of the Gaussian channel capacity, such that for any rate less than $R_{0}$, a system that yields an arbitrary small error probabilities exists [14], [15].
}

required, and is equal to

$$
\begin{gathered}
\frac{\partial\left(\sum_{k} R_{0_{k}}\right)}{\partial \boldsymbol{P}_{l}^{*}}=-\sum_{k} \\
\frac{\sum_{A, A^{\prime}} \exp \left(-\frac{p\left\|\sum_{j=1}^{K} \hat{\boldsymbol{H}}_{k j}\left(\boldsymbol{x}_{j}^{a_{j}, a_{j}^{\prime}}\right)\right\|^{2}}{4 \sigma^{2}}\right) p \hat{\boldsymbol{H}}_{l k}^{H}\left(\sum_{j=1}^{K} \hat{\boldsymbol{H}}_{k j}\left(\boldsymbol{x}_{j}^{a_{j}, a_{j}^{\prime}}\right)\right) \boldsymbol{x}_{l}^{a_{j}, a_{j}^{\prime}{ }^{H}}}{\sum_{A, A^{\prime}} \exp \left(-\frac{p\left\|\sum_{j=1}^{K} \hat{\boldsymbol{H}}_{k j}\left(\boldsymbol{x}_{j}^{a_{j}, a_{j}^{\prime}}\right)\right\|^{2}}{4 \sigma^{2}}\right)}(10)
\end{gathered}
$$

where $\boldsymbol{x}_{j}^{a_{j}, a_{j}^{\prime}}=\boldsymbol{x}_{j}^{a_{j}}-\boldsymbol{x}_{j}^{a_{j}^{\prime}}$. Tending SNR to infinity, the maximization problem of the cut-off rate becomes equivalent to the one that maximizes the minimum distance of the received constellation, which yields a problem with less complexity as can be noticed from (9).

As discussed previously, a closed form solution of the precoding matrices that maximizes the joint cut-off rate seems difficult to derive. Therefore, we propose to approach the solution iteratively. We use an iterative algorithm that optimizes one variable while the others remain fixed. Each variable is considered as one of the precoding matrices. This technique results in a non-convex optimization due to the dependence between the precoding matrices. At each iteration, the optimization is based on the gradient descent widely used in MIMO multi-user channel. The iterative algorithm is detailed as follows

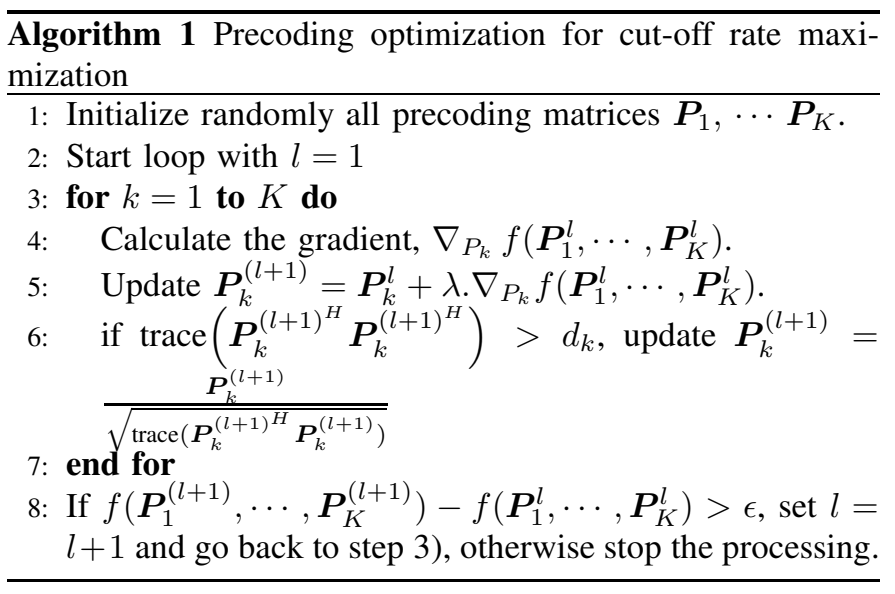
In this algorithm the gradient is defined as in (10), $f$ describes the objective function given in (9), and the precoding matrices are supposed to be of unit norm. The step size $\lambda$ is updated using the backtracking search. Despite the nonconvexity of the multivariable objective function, as long as the variable is steered in the gradient direction, the algorithm shows a convergence to a local maximum.

Remark 2: we try to maximize the joint cut-off rate rather than the cut-off rate between each transmitter and its destination, due to its simple analytic form. This entails a non-optimal performance in term of rate improvement for high SNR.

\section{Closed Form Solution of THE PRECOding MATRICES}

Motivated by the fact that high complexity level sustains the iterative algorithm for the objective functions described 
above, this section introduces a closed-form solution that tends to optimality when the SNR is close to zero. This solution is obtained using the first order Taylor expansion of the MI. In a $K$-user MIMO interference channel, the MI is derived as follows

$$
\begin{gathered}
I\left(\boldsymbol{x}_{k} ; \boldsymbol{y}_{k}\right)= \\
\log _{2}\left(M^{d_{k}}\right)+\frac{1}{M^{K d_{k}}} \sum_{a_{1}, \cdots, a_{K}} \mathbb{E}\left[\log _{2}\left(J_{1 k}\right)\right] \\
-\frac{1}{M^{K d_{k}}} \sum_{a_{1}, \cdots, a_{K}} \mathbb{E}\left[\log _{2}\left(J_{2 k}\right)\right],
\end{gathered}
$$

where

$$
\begin{gathered}
J_{1 k}=\sum_{a_{1}^{\prime}, \cdots, a_{K}^{\prime}} \exp \left(-\frac{\left\|\boldsymbol{z}_{k}+\sum_{j=1}^{K} \hat{\boldsymbol{H}}_{k j}\left(\boldsymbol{x}_{j}^{a_{j}}-\boldsymbol{x}_{j}^{a_{j}^{\prime}}\right)\right\|^{2}}{\sigma^{2}}\right), \\
J_{2 k}=\sum_{a_{1}^{\prime \prime}, \cdots, a_{K}^{\prime \prime}} \exp \left(-\frac{\left\|\boldsymbol{z}_{k}+\sum_{j \neq k}^{K} \hat{\boldsymbol{H}}_{k j}\left(\boldsymbol{x}_{j}^{a_{j}}-\boldsymbol{x}_{j}^{a_{j}^{\prime \prime}}\right)\right\|^{2}}{\sigma^{2}}\right),
\end{gathered}
$$

where $\hat{H}_{k j}=\boldsymbol{H}_{k j} \boldsymbol{P}_{j}$, and the indices $a_{j}, a_{j}^{\prime}, a_{j}^{\prime \prime}$ are in the set $\mathcal{M}_{d_{j}}=\left\{1, \cdots, M^{d_{j}}\right\}$ for all $j$ with $M$ the constellation set length for one symbol. $\boldsymbol{x}_{j}^{a_{j}}$ is a symbol vector from the $j^{t h}$ transmitter belonging to the set $\mathcal{Q}^{d_{j}}$.

In spite of the computational gain offered by the cut-off rate compared to the MI calculation, an important computational cost remains with the iterative algorithm. Motivated by the fact that the iterative algorithm suffers from high computational cost for the objective function described above, we introduce another solution in a closed-form that tends to optimality when the SNR is close to zero. This solution is obtained using the first order Taylor expansion of the MI. Assuming an SNR close to zero, using the first order Taylor expansion of $e^{U}$ and $\log (1+U)$ when $U$ is in the neighborhood of zero, and using that the constellation is zero mean at all users, the maximization problem of the total MI given in (11) can be approximated as

$$
\begin{gathered}
\underset{\boldsymbol{P}_{1}, \cdots, \boldsymbol{P}_{K}}{\arg \max } \sum_{k} I_{k}\left(\boldsymbol{x}_{k} ; \boldsymbol{y}_{k}\right) \approx \underset{\boldsymbol{P}_{1}, \cdots, \boldsymbol{P}_{K}}{\arg \max } \sum_{j} \operatorname{trace}\left(\boldsymbol{P}_{j}^{H} \boldsymbol{Q}_{j} \boldsymbol{P}_{j}\right) \\
\text { where } \boldsymbol{Q}_{j}=\boldsymbol{H}_{j j}^{H} \boldsymbol{H}_{j j} .
\end{gathered}
$$

The $d_{l}$ vectors of the precoding matrix $\boldsymbol{P}_{k}, k \in\{1, \cdots, K\}$, that maximize the function in (12) are given by

$$
\boldsymbol{P}_{k}=\nu_{\max }^{d_{l}}\left(\boldsymbol{H}_{k k}^{H} \boldsymbol{H}_{k k}\right),
$$

where $\nu_{\max }^{d_{l}}(\boldsymbol{A})$ are the eigenvectors corresponding to the $d_{l}^{t h}$ largest eigenvalues of $\boldsymbol{A}$. The solution in (13) satisfies the transmit power constraint since the precoding matrices composed of the channel matrix eigenvectors have a unit Frobenius norm.

Remark 3: the closed-form solution is characterized by its low computational complexity, and only requires at each transmitter the CSI linking it to its dedicated receiver, whereas the other solutions are achieved iteratively and require a total CSIT knowledge.

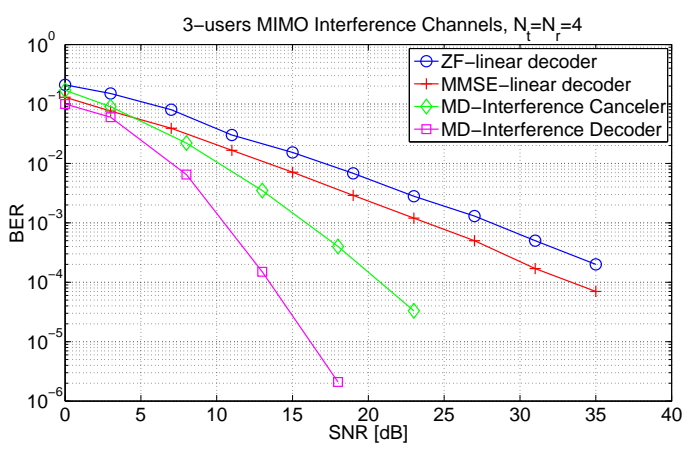

Fig. 1. BER performance comparison of the interference decoding and the traditional interference cancellation scheme

- ZF-linear decoder : the interference is canceled using $\mathrm{ZF}$, and the original signal is decoded using linear ZF decoder.

- MMSE-linear decoder: the interference is canceled using ZF, and the original signal is decoded using linear minimum mean squared error decoder.

- MD-Interference Canceler: the interference is canceled using $\mathrm{ZF}$, and the original signal is decoded using minimum-distance decoder.

- MD-Interference Decoder: the interference and the desired signal are jointly decoded using minimum-distance decoder.

\section{Simulation RESUlts}

The BER performance of the proposed solutions is evaluated in a 3-user MIMO IC. We assume an uncoded QPSK modulation for the original symbols with equal probabilities. The channel coefficients are circularly symmetric Gaussian distributed with zero mean and unit variance. Our results are based on Monte-Carlo simulations. The SNR[dB] in the figures is the SNR per antenna. The acronyms of the used designs are explained under the figures.

Fig. 1 compares the BER performance of the interference canceler using $\mathrm{ZF}$ versus the interference decoder using an MD detector when $N_{t}=N_{r}=4$ and $d_{1}=d_{2}=d_{3}=2$. For both strategies we assume an IA design at the transmitters as described in [6], and we apply the MD detector to decode the original symbols. We observe that the MD-Interference Decoder results in a steeper slope than the MD-Interference Canceler at high SNR. E.g. at BER $10^{-4}$, the MD-Interference Decoder yields a gain of $7 \mathrm{~dB}$ over the MD-Interference Canceler. This result is consistent with our expectation in Section III which says that decoding interference can achieve higher receive diversity than cancellation using ZF.

Next, Fig. 2 compares the total MI in the 3 -user $2 \times 2$ MIMO IC. We assume $d_{1}=d_{2}=d_{3}=1$. The integration in (11) is solved using Monte-Carlo integration. Comparing to the no precoding, the $\mathrm{CF}$ design shows a significant improvement, e.g. at $-5 \mathrm{~dB}$ a gain of about $0.8 \mathrm{~b} / \mathrm{s} / \mathrm{Hz}$ is obtained. This gain increases slightly until reaching $1 \mathrm{~b} / \mathrm{s} / \mathrm{Hz}$ at $5 \mathrm{~dB}$. On the other hand, compared to the P-R0 design, the CF yields a gain of about $0.3 \mathrm{~b} / \mathrm{s} / \mathrm{Hz}$ in the low to medium SNR region, and then performs closely until it reaches $10 \mathrm{~dB}$ where the P-R0 design outperforms the CF design by $0.1 \mathrm{~b} / \mathrm{s} / \mathrm{Hz}$.

Fig. 3 compares the total MI in the 3 -user $2 \times 2$ MIMO 


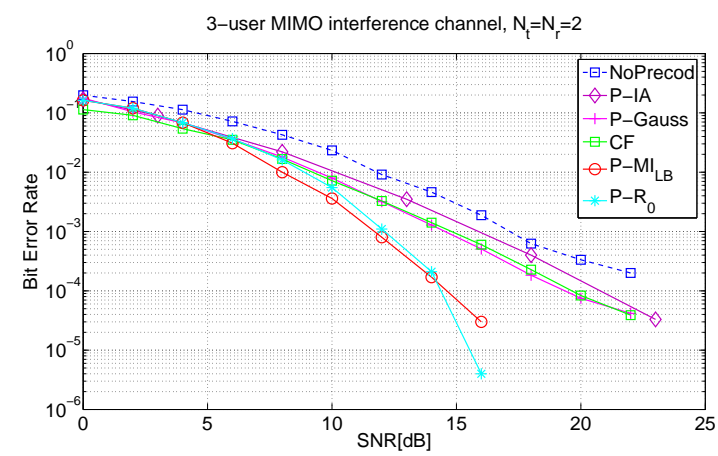

Fig. 2. BER performance comparison using different precoding design

- NoPrecod : no precoding design.

- $\mathrm{CF}$ : precoding based on the closed-form solution presented herein.

- P-IA : precoding based on the MI maximization for Gaussian inputs constellation.

- P-Gauss : precoding based on the IA concept.

- $\mathrm{P}-R_{0}:$ precoding based on the joint cut-off rate maximization.

- $\mathrm{P}-\mathrm{MI}_{L B}$ : precoding based on the MI lower bound maximization derived in [19].

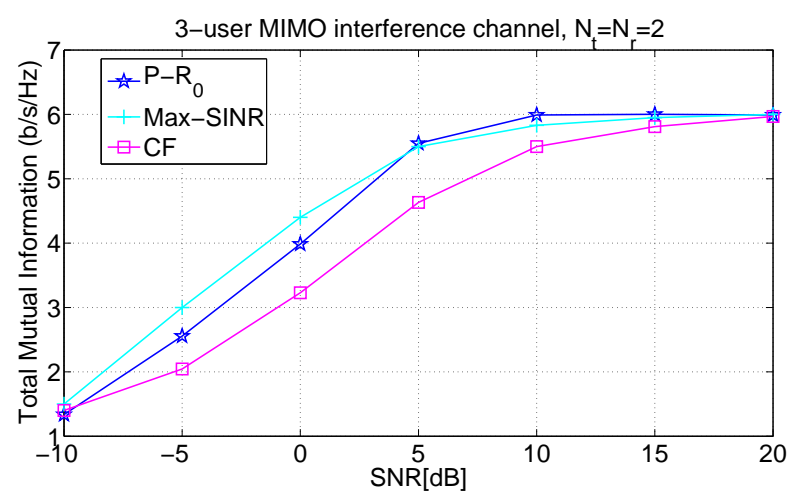

Fig. 3. Total mutual information comparison for different precoding design under BPSK modulation.

- Max-SINR: the beamforming design proposed in [6] that maximizes the SINR of all streams.

IC. We assume $d_{1}=d_{2}=d_{3}=1$. The integration in (11) is solved using Monte-Carlo method. Compared to the P-R0 design that maximizes the joint cutoff rate, a slight gain is obtained using the $\mathrm{CF}$ design and the Max-SINR design at $-10 \mathrm{~dB}$. With the SNR increases, the CF design results in lower sum-rate performance than the other precoding designs. In addition, up to $5 \mathrm{~dB}$ the Max-SINR achieves the best performance and reaches a maximum gain of $0.4 \mathrm{~b} / \mathrm{s} / \mathrm{Hz}$ compared to the P-R0 at $0 \mathrm{~dB}$. Beyond $5 \mathrm{~dB}$ and up to $20 \mathrm{~dB}$, the P-R0 outperforms the other two designs. From $20 \mathrm{~dB}$, the three designs performs the same.

\section{CONCLUSION}

In this paper, we have addressed the problem of decoding and precoding design in the $K$-user MIMO IC. We have shown that decoding interference jointly with the desired signal re- sults in an improved receive diversity order. We have proposed two precoding designs, assuming discrete constellation. One seeks for the optimum that maximizes the joint cut-off rate iteratively. The other is a closed-form obtained using first order Taylor expansion of the mutual information for low SNR. In terms of sum-rate performance, the first solution has shown an improvement over the precoding design that maximizes the SINR. In terms of BER performance, the first solution has shown a gain over all other compared schemes, especially for high SNR. The second solution has shown a remarkable gain for low and medium SNR values.

\section{REFERENCES}

[1] V. R. Cadambe and S. A. Jafar, "Interference Alignment and Degrees of Freedom of the K-user Interference Channel," IEEE Transactions on Information Theory, vol. 54, no. 8, pp. 3425-3441, Aug. 2008.

[2] M. Maddah-Ali, A. S. Motahari, and A. K. Khandani, "Communication over MIMO X Channels: Interference Alignment, Decomposition, and Performance Analysis," IEEE Transactions on Information Theory, vol. 54, pp. 3457-3470, Aug. 2008.

[3] K. LEE and D. Petersen, "Optimal Linear Coding for Vecotr Channels," IEEE Transactions on Communications, Dec. 1976.

[4] E. Telatar, "Capacity of multi-antenna gaussian channels," European Transactions on Telecommunications, vol. 10, pp. 585-595, 1999.

[5] T. Gou and S. A. Jafar, "Degrees of Freedom of the K-user NxM MIMO Interference Channel," IEEE Transactions on Information Theory, vol. 56, no. 12, Dec. 2010.

[6] K. Gomadam, V. Cadambe, and S. Jafar, "Approaching the Capacity of Wireless Networks through Distributed Interference Alignment," in Proc. of IEEE Globecom, 2008.

[7] C. Xiao, Y. Zheng, and Z. Ding, "Globally Optimal Linear Precoders for Finite Alphabets Signals Over Complex Vector Gaussian Channels," IEEE Transactions on Signal Processing, vol. 59, pp. 3301-3314, July 2011.

[8] M. Abramowitz and I. Stegun, Handbook of Mathematical Functions with Formulas, Graphs and Mathematical Tables. Dover Publications, Inc., New York, 1964.

[9] Z. Wang and G. Giannakis, "A simple and general parameterization quantifying performance in fading channels," IEEE Transactions on Communications, vol. 51, no. 8, Aug. 2003.

[10] X. Zhu and R. D. Murch, "Performance Analysis of Maximum Likelihood Detection in a MIMO Antenna System," IEEE Transactions on Communications, vol. 50, no. 2, pp. 187-191, Feb. 2002.

[11] H. Sung, S. Park, K. Lee, and I. Lee, "Linear precoder designs for k-user interference channels," IEEE Transactions on Wireless Communications, vol. 9, no. 1, pp. 291-300, Jan. 2010.

[12] Y. Fadlallah, A. Aïssa-El-Bey, K. Amis, and R. Pyndiah, "Interference Alignment: Improved Design via Precoding Vectors," in Proc. of IEEE VTC-Spring, May 2012.

[13] J. Shin and J. Moon, "Weighted Sum Rate Maximizing Linear Transceiver Filters for K-user MIMO Interference Channel," IEEE Transactions on Communications, vol. 60, pp. 2776-2783, Oct. 2012.

[14] J. Wozencraft and I. Jacobs, Principles of Communication Engineering. John Wiley and Sons, New York, 1966.

[15] A. Viterbi, "Error Bounds for Convolutional Codes and an Asymptotically Optimum Decoding Algorithm," IEEE Transactions on Information Theory, April 1967.

[16] J. Massey, "Coding and Modulation in Digital Communications," in Proc. of International Zurich Seminar, Mar. 1974.

[17] F. Rey, M. Lamarca, and G. Vaacutezquez, "Linear Precoder Design through Cut-off Rate Maximization in MIMO-OFDM," IEEE Transactions on Signal Processing, vol. 58, no. 3, 2010.

[18] J. Choi, Optimal Combining and Detection: Statistical Signal Processing for Communications. Cambridge Press, 2010.

[19] W. Zeng, C. Xiao, and J. Lu, "A Low Complexity Design of Linear Precoding for MIMO Channels with Finite Alphabet Inputs," IEEE Wireless Communications Letters, vol. 1, Feb. 2012. 